\title{
Circulation of Railway Rolling Stock: \\ A BRANCH-AND-PRICE APPROACH
}

\author{
Marc Peeters and Leo Kroon
}

\begin{tabular}{|l|l|}
\hline \multicolumn{2}{|l|}{ ERIM REPORT SERIES RESEARCH IN MANAGEMENT } \\
\hline ERIM Report Series reference number & ERS-2003-055-LIS \\
\hline Publication & 2003 \\
\hline Number of pages & 28 \\
\hline Email address corresponding author & I.kroon@fbk.eur.nl \\
\hline Address & Erasmus Research Institute of Management (ERIM) \\
& Rotterdam School of Management / Faculteit Bedrijfsunde \\
& Rotterdam School of Economics / Faculteit \\
& Economische Wetenschappen \\
& Erasmus Universiteit Rotterdam \\
& P.O. Box 1738 \\
& 3000 DR Rotterdam, The Netherlands \\
& Phone: +31 10 408 1182 \\
& Fax: +31 10 408 9640 \\
& Email: info@erim.eur.nl \\
& Internet: www.erim.eur.nl \\
\hline
\end{tabular}

Bibliographic data and classifications of all the ERIM reports are also available on the ERIM website: www.erim.eur.nl 


\title{
ERASMUS RESEARCH INSTITUTE OF MANAGEMENT
}

\author{
REPORT SERIES \\ RESEARCH IN MANAGEMENT
}

\begin{tabular}{|c|c|c|}
\hline \multicolumn{3}{|c|}{ BIBLIOGRAPHIC DATA AND CLASSIFICAT IONS } \\
\hline Abstract & \multicolumn{2}{|c|}{$\begin{array}{l}\text { We describe an algorithmic approach to determine an efficient railway rolling stock circulation on } \\
\text { a single line or on a set of interacting lines. Given the timetable and the passengers' seat } \\
\text { demand, we develop a branch-and-price algorithm hat results in an allocation of rolling stock } \\
\text { material to the daily trips. In order to efficiently utilize the train units, they can be added to or } \\
\text { removed from the trains at some stations along the line. These changes in train composition are } \\
\text { subject to several constraints, for example corresponding to the order of the train units within a } \\
\text { train. A solution is evaluated based on three criteria, i.e. the service to passengers, the } \\
\text { robustness, and the cost of the circulation. The branch-and-price algorithm that we developed is } \\
\text { tested on rea-life instances from NS Reizigers, the main Dutch operator of passenger trains. }\end{array}$} \\
\hline \multirow{3}{*}{$\begin{array}{l}\text { Library of Congress } \\
\text { Classification } \\
\text { (LCC) }\end{array}$} & $5001-6182$ & Business \\
\hline & $5201-5982$ & Business Science \\
\hline & HE 1795 & Railway operation and management \\
\hline \multirow{3}{*}{$\begin{array}{l}\text { Journal of Economic } \\
\text { Literature } \\
\text { (JEL) }\end{array}$} & M & Business Administration and Business Economics \\
\hline & $\begin{array}{l}\text { M } 11 \\
\text { R } 4\end{array}$ & $\begin{array}{l}\text { Production Management } \\
\text { Transportation Systems }\end{array}$ \\
\hline & C 61 & Optimization Techniques, Programming models; Dynamic Analysis \\
\hline \multirow{3}{*}{$\begin{array}{l}\text { European Business Schools } \\
\text { Library Group } \\
\text { (EBSLG) }\end{array}$} & $85 \mathrm{~A}$ & Business General \\
\hline & $\begin{array}{l}260 \mathrm{~K} \\
240 \mathrm{~B} \\
\end{array}$ & $\begin{array}{l}\text { Logistics } \\
\text { Information Systems Management }\end{array}$ \\
\hline & $260 \mathrm{Q}$ & Transportation \\
\hline \multicolumn{3}{|c|}{ Gemeenschappelijke Onderwerpsontsluiting (GOO) } \\
\hline \multirow[t]{3}{*}{ Classification GOO } & 85.00 & Bedrijfskunde, Organisatiekunde: algemeen \\
\hline & $\begin{array}{l}85.34 \\
85.20\end{array}$ & $\begin{array}{l}\text { Logistiek management } \\
\text { Bestuurlijke informatie, informatieverzorging }\end{array}$ \\
\hline & 85.03 & Methoden en technieken, operations reseach \\
\hline \multirow[t]{3}{*}{ Keywords GOO } & \multicolumn{2}{|c|}{ Bedrijfskunde / Bedrijfseconomie } \\
\hline & \multicolumn{2}{|c|}{ Bedrijfsprocessen, logistiek, management informatiesystemen } \\
\hline & \multicolumn{2}{|c|}{ Spoorwegen, personenvervoer, treinverkeer, organisati e } \\
\hline Free keywords & \multicolumn{2}{|c|}{$\begin{array}{l}\text { Railways, public transportation, rolling stock circulation, operations research, } \\
\text { logistics. }\end{array}$} \\
\hline
\end{tabular}




\title{
Circulation of Railway Rolling Stock: A BrANCH-AND-Price APPROACH
}

\author{
MARC PEETERS \\ Centrum voor Wiskunde en Informatica, Amsterdam. \\ Adress: NS Reizigers, Dept. of Logistics, HGB IV 9.30, P.O. Box 2025, 3500 HA Utrecht, The \\ Netherlands, \\ m.peeters@reizigers.ns.nl
}

\section{LEO KROON}

NS Reizigers, Dept. of Logistics, HGB IV 9.30, P.O. Box 2025, 3500 HA Utrecht, The Netherlands, lg.kroon@reizigers.ns.nl and Rotterdam School of Management, Erasmus University Rotterdam, P.O. Box 1738, 3000 DR Rotterdam, The Netherlands, l.kroon@fbk.eur.nl

\begin{abstract}
:
We describe an algorithmic approach to determine an efficient railway rolling stock circulation on a single line or on a set of interacting lines. Given the timetable and the passengers' seat demand, we develop a branch-and-price algorithm that results in an allocation of rolling stock material to the daily trips. In order to efficiently utilize the train units, they can be added to or removed from the trains at some stations along the line. These changes in train composition are subject to several constraints, for example corresponding to the order of the train units within a train. A solution is evaluated based on three criteria, i.e. the service to passengers, the robustness, and the cost of the circulation. The branchand-price algorithm that we developed is tested on real-life instances from NS Reizigers, the main Dutch operator of passenger trains.
\end{abstract}

Subject classification: Transportation, scheduling, vehicle: circulation of rolling stock. Programming, Integer, Applications. 


\section{Introduction}

\subsection{The Rolling Stock Circulation Problem}

The efficient circulation of railway rolling stock is an important problem for operators of passenger trains, since the rolling stock represents a huge capital investment. The rolling stock is also an investment that cannot be changed frequently, because it usually has an economic life cycle of several decades. For these reasons, it has to be decided carefully how many carriages or train units are necessary per scheduled train in order to attain a certain quality level for the passenger.

In this paper we focus on the efficient circulation of train units on a single line or train series, or on a set of interacting lines. A train series is defined by two endpoints between which several trains run up and down according to the timetable. A large part of the rolling stock is assigned to a fixed train series or a set of interacting train series. Train series interact if they have a common endpoint and if the same train units carry out trips on several of these train series sequentially. Therefore, rolling stock circulations are planned for a train series, if there is no interaction with other train series, and for a set of interacting train series otherwise. The primary objective pursued in the planning process of the rolling stock circulation is the maximization of service to the passengers by minimizing the seat shortages. Given the current timetable and the expected number of passengers, some shortages may be unavoidable, because of a limitation on the train length or because of the limited number or rolling stock material dedicated to the line. Other concerns are the robustness, as far as the allocation of rolling stock influences this, and the cost of the rolling stock circulation.

In order to obtain a better match between the available rolling stock and the passengers' seat demand, the compositions of the trains can be changed at some stations by adding or removing train units to or from the trains. For example, train units may be uncoupled from a train after the morning rush hours, and they may be coupled again before the afternoon rush, possibly onto another train. These shunting operations, which are carried out in the short time interval between the arrival of a train at a station and its subsequent departure, is subject to several practical rules related to the feasibility of the transition of the train composition.

Our approach is tested on several real-life instances of NS Reizigers, the main Dutch operator of passenger trains. The rolling stock of the train series of these instances consists of train units. Train units differ from train carriages, because they can move individually in both directions without a 
locomotive. Figure 1a presents a double deck train unit with four carriages and Figure $1 \mathrm{~b}$ presents a double deck carriage. There exist other train units of this double deck type with a different number of carriages (three carriages or six carriages), subsequently called subtypes. Such a train unit is indivisible and a train can be composed of several of those subtypes of train units. Different types of train units (e.g. double deck versus single deck), however, cannot be combined with each other in one train. It is preferable to assign only one material type to a train series or set of related train series, since in case of disruption of operations (e.g. switch or electricity breakdown), the circulation will not be completely disorganized, and it will be much easier to return to the regular situation.

Figure 1a: Double deck train unit.

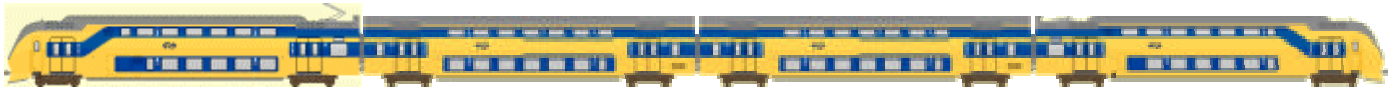

Figure 1b: Double deck carriage.

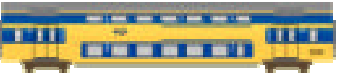

In this paper, we describe a branch-and-price approach for solving the rolling stock circulation problem for a single train series or a set of interacting train series on a single day. Although the model is also valid for supporting operational decisions related to the rolling stock circulation, the primary objective of the model is to investigate the effect of changes in some input parameters of the problem on the objectives, e.g. the number of train units assigned to a line, an additional station where units can be (un)coupled, a change in the allowable train length on some trips, etc.

\subsection{Literature Review}

In the literature, several related problems have been treated. Schrijver (1993) considers the problem of minimizing the number of train units of different subtypes for an hourly train series in the Netherlands, given that passengers' seat demand must be satisfied. The only restriction on the transition between two compositions on two consecutive trips is that the number of train units must be available at the right time and station. Other (un)coupling restrictions are ignored. Brucker, Hurink and Rolfes (1998) study the problem of finding a routing of railway carriages through a network, given a timetable. They focus on the repositioning trips of carriages from one station to another. Their solution approach is based on local search techniques like simulated annealing. Ben-Khedher, Kintanar, Queille and Stripling (1998) study the problem of allocating train units to the French High Speed Trains. Their rolling stock allocation system is based on a capacity adjustment model that is linked to the seat 
reservation system and seeks to maximize the expected profit. Cordeau, Soumis and Desrosiers (2000) present a Benders decomposition approach for the locomotive and car assignment problem. Their approach is based on the concept of train consists, i.e. a group of compatible units of equipment (locomotive(s), first and second class cars) that travel along some part of the rail network. Computational experiments show that optimal solutions can be found in short computation times. In a subsequent paper, Cordeau, Soumis and Desrosiers (2001) extend their model with various real-life constraints, as for example maintenance constraints. Lingaya, Cordeau, Desaulniers, Desrosiers and Soumis (2002) deal with the problem of assigning carriages to trains at VIA Rail in Canada. They present a model to adapt a master plan to additional information concerning the expected numbers of passengers. They allow for coupling and uncoupling of carriages at various locations in the network and explicitly take the order of the carriages in the trains into account. Several other reallife constraints, such as maintenance requirements, are considered as well. The solution approach is based on a Dantzig-Wolfe reformulation solved by column generation. Next, branch-and-bound is applied heuristically to obtain good integer solutions. Alfieri et al. (2002) describe a model to determine the circulation of rolling stock on a single line and day. Their approach is based on an Integer Programming formulation solved with a commercial IP solver and tested on real-life case-studies of the Dutch railway company. Abbink et al. (2003) present a model to allocate different material types of rolling stock to the different train series. They present an IP model, that minimizes the seat shortages during the morning rush hours by allocating material types and possibly subtypes with different capacities to all the trains running simultaneously at 8 o'clock, the busiest moment of the day. Their approach is applied to the Dutch railways for several scenarios that differ in the number of material types and subtypes that can be allocated to a line. For an excellent survey on optimization models for train routing and scheduling, we further refer to Cordeau, Toth and Vigo (1998).

In this paper, we present a branch-and-price algorithm for solving the Rolling Stock Circulation Problem. During the last decade, many hard integer programming problems have been successfully solved exactly with branch-and-price. The first papers on branch-and-price or integer programming column generation are Desrosiers, Soumis and Desrochers (1984) and Desrosiers and Soumis (1989) on routing problems. Savelsbergh (1997) presents an efficient branch-and-price algorithm for the Generalized Assignment Problem. For a general exposition on branch-and-price, we refer to Vanderbeck and Wolsey (1996), Barnhart et al. (1998) and Vanderbeck (2000). 
The Rolling Stock Circulation Problem has similarities with the Multicommodity Flow Problem (MCFP), where the flows of different commodities must be routed through a graph from supply to demand nodes without exceeding the capacities of the arcs (see e.g. Ahuja, Magnanti and Orlin, 1993). In our problem, the commodities correspond to the material subtypes, whereas the graph is determined by the different trips that must be served. The capacity of an arc corresponds to the maximum number of carriages that can be deployed on a trip, because of the limitation of the train length. Column generation approaches and branch-and-price algorithms have been proposed to tackle variants of the MCFP. For example, Barnhart, Hane and Vance (2000) present a branch-and-price-andcut algorithm to solve an origin-destination integer MCFP. Holmberg and Yuan (2003) present a column generation approach for a MCFP with side constraints. Usually, for every commodity paths through a network are generated, where the Master Problem contains arc capacity constraints. As will be explained later, we will generate paths corresponding to joint flows of several commodities through a network that meet the capacity constraints.

This paper is organized as follows. Section 2 describes the problem in more detail. Section 3 presents the model and an IP formulation, that will be used as a benchmark for our branch-and-price approach. In Section 4, we present a Dantzig-Wolfe reformulation for the problem. Section 5 outlines the branch-and-price procedure. Section 6 discusses the numerical experiments and, finally, in section 7 we conclude.

\section{Problem Description}

In this Section, we describe the problem in more detail. Section 2.1 discusses the concept of a train series and gives an example. Section 2.2 describes the feasible changes in the train compositions at the stations and Section 2.3 discusses the inventory positions of train units at the stations. Finally, section 2.4 gives the criteria on which a rolling stock circulation is evaluated.

\subsection{Train Series}

A train series is defined by two endpoints between which several trains run up and down according to the timetable. A fixed number of trains is required to run all trips of the timetable. This number is determined by the circulation time of the train series, including the return times at the endpoints, and its frequency. For example, Figure 2 presents the railway map of the Netherlands. The bold line presents the intercity 2100 train series running between Amsterdam (Asd) and Vlissingen (Vs), the 2400 
between Asd and Dordrecht (Ddr), and the 2600 between Asd and The Hague (Gvc). They interact with each other, because, after a 2600 train has made his journey from Asd to Gvc and back, its train number changes and it will perform a roundtrip to Vs (2100) or Ddr (2400). The sequence is not arbitrary. In fact, if a 2100, 2400 or 2600 train arrives in Asd, its next journey will be the first 2100 , 2400 or 2600 roundtrip that leaves Asd after its arrival. The cyclic nature of the timetable results in the same sequence of roundtrips, presented in Table 1. The last two columns present the arrival and departure time for the trips from and to Asd. After the fourth roundtrip, a train will carry out the first roundtrip again. The total time, including the waiting time, for the four roundtrips is 14 hours. Therefore, at least 14 trains are required to run the timetable for the 2100, 2400 and 2600 train series. As illustrated by Table 1, we see that after 14 hours the train is back in Amsterdam and ready to carry out the first roundtrip again. Due to some deviations from the regular pattern during the morning rush hour, even 15 trains are required.

Figure 2: Train series 2100, 2400, 2600.

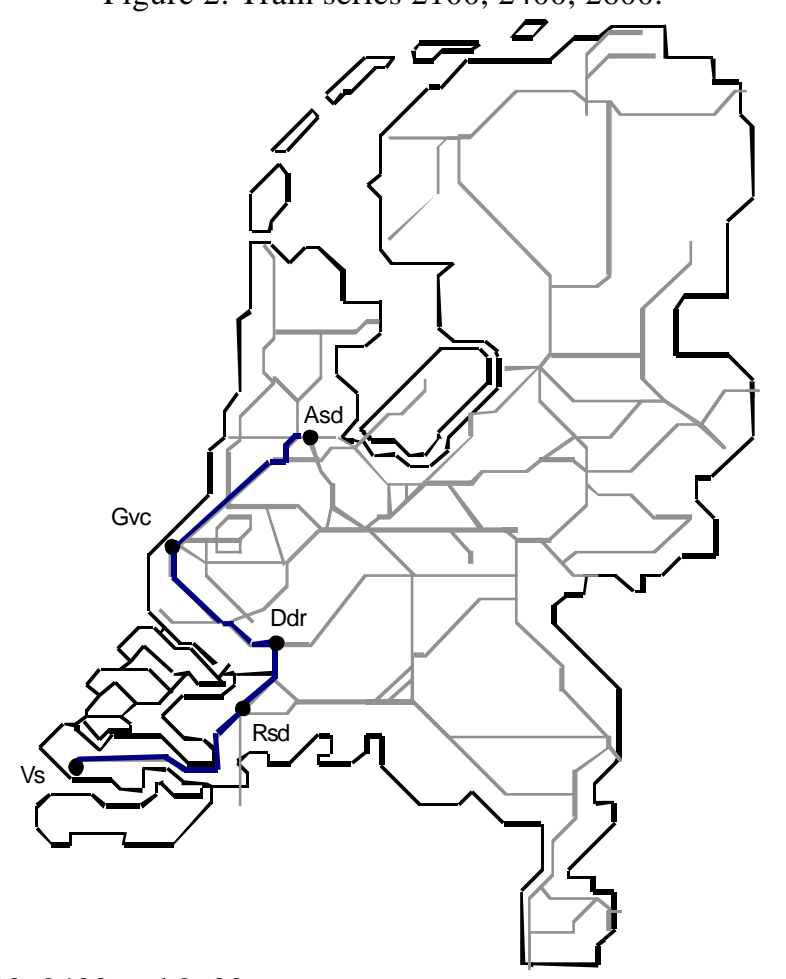

Table 1: Train series 2100, 2400 and 2600.

\begin{tabular}{lccrrrr}
\hline \hline & Roundtrip & Trainnumber & Asd - Xyz & \multicolumn{1}{c}{ Xyz - Asd } \\
\hline 1 & Asd-Gvc-Asd & 2600 & $x \mathrm{~h} 13-(x+1) \mathrm{h} 03$ & $(x+1) \mathrm{h} 29-$ & $(x+2) \mathrm{h} 20$ \\
2 & Asd-Ddr-Asd & 2400 & $(x+2) \mathrm{h} 29-(x+3) \mathrm{h} 47$ & $(x+4) \mathrm{h} 13-$ & $(x+5) \mathrm{h} 33$ \\
3 & Asd-Gvc-Asd & 2600 & $(x+5) \mathrm{h} 43-(x+6) \mathrm{h} 33$ & $(x+6) \mathrm{h} 59-$ & $(x+7) \mathrm{h} 50$ \\
4 & Asd-Vs-Asd & 2100 & $(x+7) \mathrm{h} 59-(x+10) \mathrm{h} 36$ & $(x+11) \mathrm{h} 26-(x+14) \mathrm{h} 03$ \\
\hline
\end{tabular}

As is illustrated by the example, a train makes several consecutive trips during the day. In the dense Dutch railway timetable, the sequence of the trips is fixed and input to the model. A trip is 
characterized by a departure/arrival time/station. In both the arrival and departure station, coupling and uncoupling can take place. On a trip, the train can make intermediate stops at other stations, but those are only relevant if the train composition can be changed. In the above example, the train composition can be changed at the intermediate station Roosendaal (Rsd) on the trip Asd-Vs and Vs-Asd. Therefore those trips are divided in two trips Asd-Rsd and Rsd-Vs or Vs-Rsd and Rsd-Asd. On the contrary, a stop in Rotterdam is not relevant, since a change in train composition is not allowed here. Table 2 presents the consecutive trips that one of the 15 trains must make during a single day. The first two columns give the departure and arrival time, next the train number, the departure and arrival station.

Finally, for every trip, the required capacity of first and second class passengers seats is determined as follows. Every trip is divided in subtrips, corresponding to the intermediate stops that the train makes. For example, between Asd and Gvc, the 2600 train stops at Schiphol (Shl) and Leiden (Ledn), resulting in three subtrips, i.e. Asd-Shl, ShlLedn, Ledn-Gvc. For these subtrips, first and second class passengers' counts of the conductors are known. Those counts are then changed into the required capacity by a statistical procedure, which falls outside the scope of this paper.

Table 2: Consecutive trips of a 2100-2400-2600 train.

\begin{tabular}{rrrrr}
\hline \hline$t_{s}$ & $t_{a}$ & $N r$ & $d s$ & $a s$ \\
\hline $7 \mathrm{~h} 26$ & $8 \mathrm{~h} 17$ & 2122 & $\mathrm{Vs}$ & Rsd \\
$8 \mathrm{~h} 20$ & $10 \mathrm{~h} 03$ & 2122 & Rsd & Asd \\
$10 \mathrm{~h} 13$ & $11 \mathrm{~h} 03$ & 2641 & Asd & Gvc \\
$11 \mathrm{~h} 29$ & $12 \mathrm{~h} 20$ & 2630 & Gvc & Asd \\
$12 \mathrm{~h} 29$ & $13 \mathrm{~h} 47$ & 2449 & Asd & Ddr \\
$14 \mathrm{~h} 13$ & $15 \mathrm{~h} 33$ & 2444 & Ddr & Asd \\
$15 \mathrm{~h} 43$ & $16 \mathrm{~h} 33$ & 2663 & Asd & Gvc \\
$16 \mathrm{~h} 59$ & $17 \mathrm{~h} 50$ & 2652 & Gvc & Asd \\
$17 \mathrm{~h} 59$ & $19 \mathrm{~h} 42$ & 2171 & Asd & Rsd \\
$19 \mathrm{~h} 45$ & $20 \mathrm{~h} 36$ & 2171 & Rsd & Vs \\
$21 \mathrm{~h} 26$ & $22 \mathrm{~h} 17$ & 2178 & Vs & Rsd \\
$22 \mathrm{~h} 20$ & $0 \mathrm{~h} 03$ & 2178 & Rsd & Asd \\
$0 \mathrm{~h} 13$ & $1 \mathrm{~h} 03$ & 2697 & Asd & Gvc \\
\hline
\end{tabular}

\subsection{Train Compositions}

The available rolling stock for a train series usually consists of the same material type with different subtypes. A train can be composed of several train units of different subtypes, subsequently called a composition. Those subtypes differ in number of carriages and, as a res ult, in capacity, measured in numbers of first and second class passengers' seats. A train unit can be coupled to or uncoupled from a composition at the end of a trip. The position where units can be coupled to or uncoupled from the train is fixed for every station, i.e. at the rear or the front side of a train. In a terminal station, i.e. a station 
where trains arrive from and leave to the same direction (e.g. Vs) coupling and uncoupling usually occurs at the station side of the train. In the station Rsd, where a train comes from the direction Vs and leaves in the direction Asd (or vice versa) a unit is coupled onto the front of an incoming train and uncoupled from the rear of an outgoing train. Therefore, not only the number of train units of each subtype, or the combination, is important, so is the order of the units in the composition, or the permutation. In addition, only one shunting operation for the same train can be performed at a station, i.e. coupling units to or uncoupling units from the train but not both, because the time that a train stays at a station is too short to allow for several shunting operations. Therefore, it is not possible to remove a unit in the middle of the composition from the train. Finally, the model must also take into account that if a train enters and leaves the station in the same direction, the composition of the train is turned around, i.e. the front unit becomes the rear one and vice versa. This occurs at the endpoints Asd, Ddr, Gvc and Vs.

\subsection{Inventory of Train Units at the Stations}

The limited availability of rolling stock causes interaction between the different trains. The initial inventory of train units must be divided over the stations. A train unit can only be coupled onto an incoming train, if it is available in the right station and at the right moment in time. If a train unit is uncoupled, it is not immediately available to be coupled onto another train. A certain amount of "shunting time" must be taken into account. We further assume that a train unit is uncoupled at the moment that the train arrives in the station and a unit is coupled at the moment that the train departs from the station. Therefore, we determine the local shunting time at a station such that the arrival time increased with the shunting time of train $A$ at the station is smaller than the departure time of train $B$ at the station, if train $B$ is the first train after the arrival of train $A$ to which uncoupled train units from train $A$ can be coupled. On the other hand, the arrival time of train $A$ increased with the shunting time must be greater than the departure times of all trains leaving the station earlier than train $B$.

Figure 3 presents the Time-Space Diagram for the time window from $9 \mathrm{~h} 00$ to $12 \mathrm{~h} 00$, focusing on the trips arriving in and leaving from Rsd. The straight lines represent the trips, whereas the dotted arrows present the shunting time and indicate the moment in time that an uncoupled train unit becomes available to be coupled onto another train. The 2101 train for example leaves Vs at $9 \mathrm{~h} 26$ and arrives in Rsd at 10h17. It proceeds its journey to Asd 3 minutes later at 10h20. Between 10h17 and 10h20, train units can be uncoupled from the train, but they are not immediately available to be coupled onto 
another train, due to the shunting time of 30 minutes in this example. This is reflected by the dotted arrow. Therefore, an uncoupled unit from the 2101 train cannot be coupled to the 2102 train, coming from Asd and going to Vs at $10 \mathrm{~h} 45$.

Figure 3: Time-space diagram: Vs-Rsd-Asd.

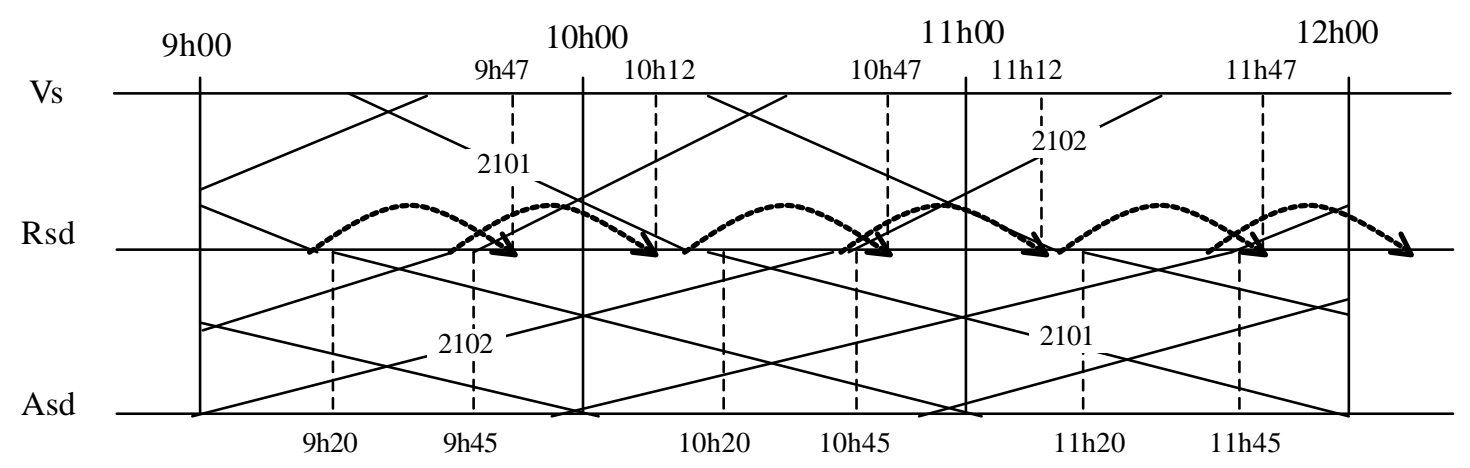

Finally, it is often assumed that the rolling stock circulation must be cyclic over a certain period of time. A way of taking care of the cyclicity requirement, is imposing that the begin inventory at all stations for every material subtype must equal the end inventory. However, this requirement is not very stringent, certainly not if the time period is short (e.g. one day). In fact, a good circulation should be such that at the end of the day, the train units end in such stations that the trips on the next day can be served appropriately, and therefore exact cyclicity is not required.

\subsection{Evaluation Criteria}

A circulation is evaluated based on three criteria, the kilometer-shortages, the number of shunting operations, and the carriage-kilometers. Given the capacity of first and second class passengers of the various subtypes of rolling stock and the composition of the train, the shortages on each subtrip can be computed. Those are multiplied by the length of the subtrip, and taking the sum over all subtrips results in kilometer-shortages for the entire trip. The second objective is minimizing the shunting operations, i.e. the number of times units must be (un)coupled to (from) the train. Those operations can cause delays, and since punctuality or robustness is an important concern, their number should be controlled. The third objective is minimizing the total number of carriage-kilometers, which are used as an approximation for the variable rolling stock cost. Carriage-kilometers are positively correlated to power supply (electricity or diesel), but also to the maintenance of the rolling stock. After a certain number of kilometers, each train unit is directed to a maintenance station for a preventive check-up and possibly for a repair. Therefore, the maintenance cost is correlated with the number of train unit or carriage- 
kilometers. Moreover, a decrease in the number of train unit or carriage-kilometers does not only lead to a decrease in maintenance cost, but also to a decrease in the maintenance requirements. Less maintenance may lead to an increase in the effectively available rolling stock capacity.

Since especially the first and second criterion cannot be expressed in an amount of money, we use weighting factors for the three criteria. By varying these weights, we obtain alternative solutions, which can be evaluated by the management of the railway operator or by the planners.

\section{Model Description}

\subsection{Transition Graph}

For each train, the possible changes in its composition at a station can be represented by a so-called transition graph, where the nodes represent the feasible train compositions on a trip and the arcs represent the feasible transitions between compositions at a station. Transition graphs were used also by Alfieri et al. (2002). Each train starts with an artificial trip from the depot at the departure station to the platform and ends with a trip to the depot at the arrival station of its last trip. The composition on these trips is the " 0 "-composition, i.e. all train units deployed on the first trip must be coupled to the train and removed from the inventory at the departure station, and all units deployed on the last trip must be added to the inventory at the final arrival station. For each trip, the expected numbers of first and second class passengers are given. Due to safety regulations, the expected shortage of passengers' seats should not exceed a certain percentage of the available seats. In addition, there is a limit on the total length of the train due to the platform lengths of the stations where the train makes intermediate stops. Therefore, many possible compositions can be excluded a priori.

Figure 4 presents a transition graph of five trips for a train of the 2100-2400-2600 train series. The train compositions are presented in the format " $\mathrm{U}_{r} \mathrm{U}_{r-1} \ldots \mathrm{U}_{1}$ ", where $\mathrm{U}_{r}$ is the train unit at the rear of the train, $\mathrm{U}_{r-1} \ldots \mathrm{U}_{2}$ are the train units in the middle of the train, and $\mathrm{U}_{1}$ is the train unit in front of the train. In this example, we assume that only train units of 4 and 6 carriages are available. The total length of the train cannot exceed 12 carriages and between Rsd and Vs the limit is just 9 carriages. The first real trip runs from Gvc to Asd and the possible compositions are given in Figure 4. If composition "4" is used on this trip, then in Asd the composition can change to "44", "64" or "444". The compositions "4" and "6" are not allowed on the next trip between Asd and Rsd, because it does not have the required number of seats. Observe that the train composition is turned around in Asd such that 
composition "64" becomes "46". On the trip Rsd-Vs, only three compositions are possible, i.e. "4","6" and "44", because the total length may not exceed 9 carriages. Suppose now that on this last trip composition " 4 " cannot be used, because the expected number of passengers is too high, then the composition "64" on the trip Asd-Rsd can be excluded as well, since it has no follower. The same holds then for composition "46" on Gvc-Asd. Next the train returns to Rsd and then to Asd, where composition " 4 " cannot be used because the expected number of passengers is too high.

Figure 4: Transition graph.

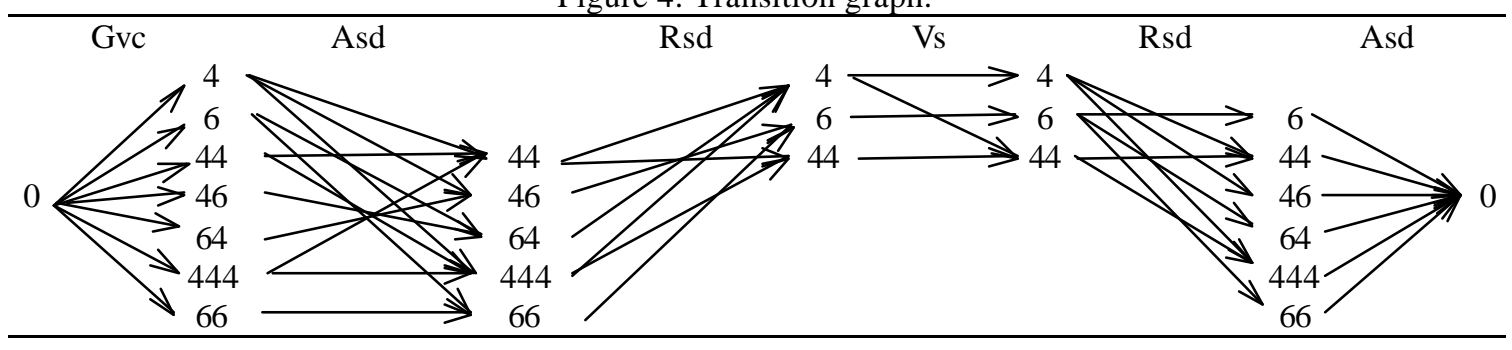

\subsection{Inventory of Train Units}

For each train, the model must select a composition for every trip. This can be considered as selecting a path in the transition graph from the " 0 "-node of the artificial first trip to the "0"-node of the artificial last trip. Since a train unit can only be coupled onto a train if it is available at the right time and station, the interaction between the different trains that run simultaneously is modeled by keeping track of the "inventory" position of each material subtype at all relevant events at the stations during the considered time period. The inventory position at an event $e$ equals the initial allocation of rolling stock material to the station augmented with the uncoupled train units and decreased with the coupled units before event $e$. The model must make sure that at any time, in all stations, the inventory positions of all material subtypes are non-negative. To this end, we determine the set of relevant events at the stations as follows. For every trip, we distinguish two events at the arrival station:

(i) A $\Delta^{-}$-event, corresponding to a potential decrease in the inventory position at the arrival station, and with the departure time of the following trip as realisation time.

(ii) $\mathrm{A} \Delta^{+}$-event, corresponding to a potential increase in the inventory position at a station and thus with the arrival time increased with the local shunting time as realisation time.

The latter is required, because during the shunting operation the train unit can not be coupled onto another train and should not immediately be added to the inventory at the station. After enumerating all events at a station, we sort them in increasing order of realisation time. The relevant events are then the $\Delta^{-}$-events that are immediately followed by a $\Delta^{+}$-event. A $\Delta^{+}$-event as such is not relevant, since the 
previous inventory position must be non-negative and a $\Delta^{+}$-event can only increase the available rolling stock. As a result, it can never violate the condition that the inventory must be non-negative. If several $\Delta^{-}$-events follow each other, then only the last $\Delta^{-}$-event is relevant. If after the last $\Delta^{-}$-event the inventory position is still non-negative, the latter holds for the $\Delta^{-}$-events immediately preceding the last $\Delta^{-}$-event as well. In the Time-Space Diagram of Figure 3, the realisation times of the $\Delta^{-}$events are indicated below the diagram $(9 \mathrm{~h} 20,9 \mathrm{~h} 45, \ldots, 11 \mathrm{~h} 45)$ and of the $\Delta^{+}$-event above the diagram $(9 \mathrm{~h} 47,10 \mathrm{~h} 12, \ldots)$. The relevant events are then the $\Delta^{-}$-events at $9 \mathrm{~h} 45,10 \mathrm{~h} 45$ and $11 \mathrm{~h} 45$.

Finally, we note that train units, entering and leaving a station being part of the same train, are not added to, nor removed from the inventory at the station at any time. We could add them upon arrival of the train and remove the same units when leaving again from the station. However, since these units do really not affect the inventory position, we ignore this temporary increase in inventory.

\subsection{Mathematical Formulation}

In order to formulate the rolling stock circulation problem, we use the following notation:

T: $\quad$ set of trips, index $t$;

$T_{0}$ : $\quad$ set of trips leaving from a depot, i.e. trips without a predecessor;

$p(t)$ : trip preceding trip $t \in T \backslash T_{0}$

MT: $\quad$ set of different material subtypes, index $l$;

$N^{l}: \quad$ the available stock of subtype $l \in M T$;

$K_{t}: \quad$ set of possible compositions for trip $t \in T$, indices $j$ or $k$;

$P_{t, k}: \quad$ set of possible preceding compositions if composition $k \in K_{t}$ is used on trip $t \in T$;

$F_{t, k}: \quad$ set of possible following compositions if composition $k \in K_{t}$ is used on trip $t \in T$;

$S: \quad$ set of stations, index $s$;

$E_{s}: \quad$ set of relevant events at station $s$, i.e. the initial state " 0 ", a $\Delta^{-}$-event, if it is immediately followed by a $\Delta^{+}$-event, and the final state " $f$ ", index $e$;

$q(e)$ : relevant event at station $s$ immediately preceding event $e \in E_{s}$.

In our model, we use two types of variables. First, we associate a variable with every arc in the transition graphs of the trains, indicating whether or not the arc is used. More specifically, for every trip $t$, composition $k \in K_{t}$ and following composition $j \in F_{t, k}$, a variable $x_{t, k, j}$ is defined, which 
equals 1 , if composition $k$ is used on trip $t$ and $j$ on the next trip, and 0 otherwise. With every variable, a cost parameter $c_{t, k, j}$ is associated, which equals the weighted kilometer-shortage cost, carriagekilometer cost if composition $k$ is used on trip $t$, and the shunting cost if composition $k$ differs from composition $j$ on the next trip. The shortage cost and kilometer cost of using composition $j$ on the next trip are not included in the cost $c_{t, k, j}$, but in the cost associated with the arcs leaving nodes corresponding to the next trip. Finally, in order to establish the link between the arc variables and the inventory positions at the stations, we associate with every variable two parameters, indicating the number of units of a material type that is (un)coupled:

$a_{t, k, j}^{l}$ : the number of uncoupled units of material subtype $l$ at the end of trip $t$, given that composition $k$ changes to composition $j$;

$b_{t, k, j}^{l}:$ the number of coupled units of material subtype $l$ at the end of trip $t$, given that composition $k$ changes to composition $j$.

For the trips $t \in T_{0}$, the number of coupled train units $b_{t, k, j}^{l}$ of material subtype $l$ equals all the units of the composition $j$, because the composition $k$ on the artificial trip is the " 0 "'-composition (see Figure 4). The number of uncoupled units for every material subtype $l$ of a final trip $t$ to the depot $a_{t, k, j}^{l}$ equals all units of composition $k$, since composition $j$ is again the " 0 "-composition.

A second type of variables consist of inventory variables at the stations. With every relevant event $e \in E_{s}$ at station $s$ and for every material type $l \in M T$, we associate a decision variable $I N V_{e, s}^{l}$, indicating the number of units of type $l$ at station $s$ after event $e$. In order to model the changes in the inventories of the material subtypes at a station $s$ between two relevant events, $q(e)$ and $e \in E_{s}$, we must determine the set of trips corresponding to a $\Delta^{-}$-event, causing a decrease in the inventory, and the set of trips corresponding to a $\Delta^{+}$-event, causing an increase in the inventory. Therefore, let $r t_{e}$ be the realisation time of relevant event $e$, then we can define both sets, as follows:

$A_{e, s}: \quad$ set of trips, arriving in $s$, for which the arrival time increased with the shunting time at station $s$ belongs to the interval $\left(r t_{q(e)}, r t_{e}\right]$ with $e \in E_{s}$;

$D_{e, s}: \quad$ set of trips, arriving in $s$, for which the departure time of the following trip at station $s$ belongs to the interval $\left(r t_{q(e)}, r t_{e}\right]$ with $e \in E_{s}$; 
Observe that the set $D_{e, s}$ does not contain the departing trips from station $s$, but trips arriving in station s. In order to determine whether or not a particular trip belongs to the set, however, we use the departure time of the next trip, since this is the realisation time of a $\Delta^{-}$-event.

In the example of Figure 3, we recall that the relevant events at the station Rsd occur at 9h45, $10 \mathrm{~h} 45$ and $11 \mathrm{~h} 45$, and we consider the time interval $(10 \mathrm{~h} 45,11 \mathrm{~h} 45]$. Then, the set $D_{e, s}$ consists of two trips: (1) Vs-Rsd arriving in Rsd at $11 \mathrm{~h} 17$ and (2) Asd-Rsd, arriving in Rsd at 11h42. The set $A_{e, s}$ consists of two trips as well: (1) Vs-Rsd arriving in Rsd at 10h17 and (2) Asd-Rsd, arriving in Rsd at 10h42. Now, we can formulate the problem as follows:

(i) Minimize the cost;

$$
\operatorname{Min} \sum_{t \in T} \sum_{k \in K_{t}} \sum_{j \in F_{t, k}} c_{t, k, j} x_{t, k, j}
$$

Subject to

(ii) Flow conservation constraints;

$$
\begin{array}{ll}
\sum_{k \in K_{t}} \sum_{j \in F_{t k}} x_{t, k, j}=1 & \forall t \in T_{0} \\
\sum_{j \in P_{t, k}} x_{p(t), j, k}=\sum_{j \in F_{t, k}} x_{t, k, j} & \forall t \in T \backslash T_{0}, \forall k \in K_{t}
\end{array}
$$

(iii) Initial inventory;

$$
\sum_{s \in S} I N V_{0, s}^{l}=N^{l} \quad \forall l \in M T
$$

(iv) Inventory constraints;

$$
\begin{gathered}
I N V_{q(e), s}^{l}+\sum_{t \in A_{e, s}} \sum_{k \in K_{t}} \sum_{j \in F_{t, k}} a_{t, k, j}^{l} x_{t, k, j}-\sum_{t \in D_{e, s}} \sum_{k \in K_{t}} \sum_{j \in F_{t, k}} b_{t, k, j}^{l} x_{t, k, j}-I N V_{e, s}^{l}=0 \\
\forall s \in S, \forall l \in M T, \forall e \in E_{s} \backslash\{0\}
\end{gathered}
$$

(v) Cyclicity constraints;

$$
I N V_{0, s}^{l}=I N V_{f, s}^{l} \quad \forall s \in S, \forall l \in M T
$$

(vi) Non-negativity and integrality;

$$
\begin{array}{ll}
x_{t, k, j} \in\{0,1\} & \forall t \in T, \forall k \in K_{t}, \forall j \in F_{t, k} \\
I N V_{e, s}^{l} \geq 0 & \forall s \in S, \forall l \in M T, \forall e \in E_{s}
\end{array}
$$


Constraints (2) impose that for every trip without a predecessor exactly one arc must be chosen, resulting in a composition for the first trip and the transition in composition at the arrival station of the first trip. Constraints (3) are flow conservation constraints, i.e. for a node in the transition graph characterized by a trip $t$ and a composition $k \in K_{t}$, the incoming flow, which equals the sum of the flows of the arcs arriving in the node, must be equal to the outgoing flow. Constraints (4) impose that the sum of the initial inventories at the stations must be equal to the total availability $N^{l}$ for each material subtype $l \in M T$. Constraints (5) are the balancing constraints, stating that the inventory of material subtype $l \in M T$ at station $s \in S$ of the previous event increased with the number of uncoupled train units in the time period between event $q(e)$ and event $e$ and decreased with the number of coupled train units of subtype $l$ in the time period between event $q(e)$ and event $e$ must be equal to the inventory at event $e$. The cyclicity constraints (6) impose that the initial inventory at a station must be equal to the end inventory for every material subtype. Finally, constraints (7) specify the integrality condition on the arc variables, whereas constraints (8) reflect the non-negativity condition for the inventory positions at the stations.

\section{Dantzig-Wolfe Decomposition}

\subsection{Formulation}

The rolling stock circulation problem (1)-(8) is suitable to apply Dantzig-Wolfe decomposition (Dantzig and Wolfe, 1960). In contrast to multicommodity flow decomposition approaches in the literature (e.g. Barnhart et al., 2000, or Holmberg and Yuan, 2003), where paths through the network are generated for each commodity and the capacity constraints are considered in the master problem, we decompose the problem based on the trains. Each train can be composed of several commodities, i.e. the material subtypes. In the Dantzig-Wolfe reformulation, we associate a variable with each path through the transition graph of all trains. The number of trains equals the number of trips without predecessor, or in the set $T_{0}$. To state the Dantzig-Wolfe reformulation, we need the following additional notation:

$R: \quad$ set of trains, index $i$;

$\Omega^{i}: \quad$ set of paths through the transition graph of train $i \in R$, index $\omega$;

$T^{i}: \quad$ set of trips corresponding to train $i$. 
Again, we introduce two types of variables, i.e. (i) the inventory variable $I N V_{e, s}^{l}$ for every relevant event $e \in E_{s}$ at station $s$ and for every material type $l \in M T$ as defined before, and (ii) the path variable $\lambda_{\omega}^{i}$, which equals 1 , if path $\omega \in \Omega^{i}$ is selected in the solution, and 0 otherwise. The cost associated with path $\omega \in \Omega^{i}$, denoted as $\chi_{\omega}^{i}$, equals the cost of the arcs used by the path. Finally, with every path $\omega \in \Omega^{i}$, we associate a set of parameters $q_{t, k, j}^{\omega}$ for every trip $t \in T^{i}$ and for every composition $k \in K_{t}$ and following composition $j \in F_{t, k}$. These parameters are associated with the arcs in the transition graph for the train $i(t)$ covering trip $t$, and describe the path $\omega \in \Omega^{i}$. Therefore, the parameter $q_{t, k, j}^{\omega}$ equals 1 , if path $\omega$ uses the corresponding arc in the transition graph, and 0 otherwise. Now the Dantzig-Wolfe reformulation can be stated as follows:

(i) Minimize the cost;

$$
\operatorname{Min} \sum_{i \in R} \sum_{\omega \in \Omega^{i}} \chi_{\omega}^{i} \lambda_{\omega}^{i}
$$

Subject to

(ii) Select a path for every train;

$$
\sum_{\omega \in \Omega^{i}} \lambda_{\omega}^{i}=1 \quad \forall i \in R
$$

(iii) Initial inventory constraints (4);

(iv) Inventory constraints;

$$
\begin{gathered}
I N V_{q(e), s}^{l}+\sum_{t \in A_{e, s}} \sum_{\omega \in \Omega^{i(t)}} \sum_{k \in K_{t}} \sum_{j \in F_{t, k}} a_{t, k, j}^{l} q_{t, k, j}^{\omega} \lambda_{\omega}^{i(t)}-\sum_{t \in D_{e, s}} \sum_{\omega \in \Omega^{i(t)}} \sum_{k \in K_{t}} \sum_{j \in F_{t, k}} b_{t, k, j}^{l} q_{t, k, j}^{\omega} \lambda_{\omega}^{i(t)}-I N V_{e, s}^{l}=0 \\
\forall s \in S, \forall l \in M T, \forall e \in E_{s} \backslash\{0\}
\end{gathered}
$$

(v) Cyclicity constraints (6);

(vi) Non-negativity and integrality (8);

$$
\lambda_{\omega}^{i} \in\{0,1\} \quad \forall i \in R, \forall \omega \in \Omega^{i}
$$

Constraints (10) impose that for every train a path must be selected and are known as the convexity constraints. Constraints (11) replace the original inventory constraints (5). In general, this formulation has a huge number of path variables, and it is impossible to enumerate them all. Therefore, we solve the LP relaxation of problem (4), (6), (9)-(12) using column generation as explained in the next section. 


\subsection{Solving the LP Relaxation}

The problem (4), (6), (9)-(11) is initialized with a small subset of the columns from the sets $\Omega^{i}, i \in R$ and is called the restricted master problem. This problem is then solved using the simplex algorithm. Based on the dual prices corresponding to constraints (11), denoted as $\pi_{e, s}^{l}$, new columns are generated from the sets of paths $\Omega^{i}, \quad i \in R$. Our goal is to find a path with minimum reduced cost, for each train $i \in R$. The reduced cost consists of four components, namely (i) the shortage cost, (ii) the shunting cost and (iii) the carriages kilometer cost, as explained before, and (iv) the material cost or revenue. Indeed, the dual prices $\pi_{e, s}^{l}$ can be interpreted as the value of a unit of material subtype $l$ in station $s$ during the time interval $\left(r t_{q(e)}, r t_{e}\right]$, i.e. if for a train that departs from station $s$ during the time interval $\left(r t_{q(e)}, r t_{e}\right], b$ units of material type $l$ are coupled to the train, then an additional cost of $b \times \pi_{e, s}^{l}$ is incurred. On the other hand, if $a$ units of material type $l$ are uncoupled from the train and added to the inventory during the period $\left(r t_{q(e)}, r t_{e}\right]$, then a revenue is received of $a \times \pi_{e, s}^{l}$.

More formally, for train $i$, the pricing problem consists of finding the shortest path through the transition graph of train $i$. The costs of the arcs are modified based on dual price information of the last solved restricted master problem. Let $s a(t)$ be the arrival station of trip $t$, and $e a(t)$ the first relevant event after the $\Delta^{+}$-event of the trip in the station, i.e. $t \in A_{e a(t), s a(t)}$. Similarly let $e d(t)$ be the relevant event at the arrival station of the trip after or corresponding to the $\Delta^{-}$-event of the trip, i.e. $t \in D_{e d(t), s a(t)}$. Then the pricing problem for train $i$ can be stated as follows:

Minimize the reduced cost;

$$
z_{P P}^{i}=\operatorname{Min} \sum_{t \in T^{i}} \sum_{k \in K_{t}} \sum_{j \in F_{t, k}}\left(c_{t, k, j}-\sum_{l \in M T}\left(\pi_{e a(t), s a(t)}^{l} a_{t k j}^{l}-\pi_{e d(t), s a(t)}^{l} b_{t, k, j}^{l}\right)\right) x_{t, k, j}
$$

Subject to

$$
\begin{array}{ll}
\sum_{k \in K_{t}} \sum_{j \in F_{t k}} x_{t, k, j}=1 & \forall t \in T_{0} \cap T^{i} \\
\sum_{j \in P_{t, k}} x_{p(t) j k}=\sum_{j \in F_{t, k}} x_{t k j} & \forall t \in T^{i} \backslash T_{0}, \forall k \in K_{t}
\end{array}
$$

Observe that problem (13)-(15) is a shortest path problem. As a result, it can be solved in polynomial time. Columns are added to the master problem as long as columns with negative reduced cost exist, 
i.e. $z_{P P}^{i}-\rho_{i}<0$, where $\rho_{i}, i \in R$ denotes the dual price of the convexity constraint (10). However due to numerical instability, it may occur that columns price out, i.e. have a negative reduced cost, although they are already included in the restricted master problem and as a result convergence is not assured. To cope with this problem, we use a somewhat different stopping criterion. At each column generation iteration, we solve the pricing problem (13)-(15) for every train. Let $\bar{Z}_{L P}$ be the optimal LP objective function value of the restricted master problem, and $Z_{L P}$ the optimal LP value of model (4), (6), (9)(11), then the following inequality holds (see e.g. Wolsey, 1998),

$$
\bar{Z}_{L P} \geq Z_{L P} \geq \bar{Z}_{L P}+\sum_{i \in R}\left(z_{P P}^{i}-\rho_{i}\right)
$$

As a result, at each iteration, we have a lower and upper bound on the optimal LP objective function value $Z_{L P}$. We stop generating columns, if the relative difference between the lower and upper bound is smaller than $\varepsilon$, where $\varepsilon=5 \times 10^{-5}$ or

$$
\sum_{i \in R}\left(z_{p p}^{i}-\rho_{i}\right) /\left(\bar{Z}_{L P}+\sum_{i \in R}\left(z_{p p}^{i}-\rho_{i}\right)\right) \leq \varepsilon
$$

As long as condition (17) is not met, the pricing problem is solved for every train and we look for the generated column with the most negative reduced cost over all trains, denoted as $r c^{*}$. Next, we add all generated columns to the restricted master for which $z_{P P}^{i}-\rho_{i}<\delta \times r{ }^{*}$, where $\delta$ has the value 0.01 . We do this in order to avoid the possibility that columns with a slightly negative reduced cost are added to the restricted master problem, if stopping criterion (17) is not met yet, because for other trains new columns with a relatively high negative reduced cost still price out. As a result, the procedure is not sensitive to the choice of the parameter $\delta$.

Finally, it is well-known that columns generated in earlier stages of the column generation procedure are often of poor quality, i.e. although their reduced cost is low, their true cost is high. This is because the initial dual prices are far from the optimal dual prices. Therefore, a "good" initialization of the restricted master problem is useful. However, we propose a two phase procedure to cope with this issue. In a first phase, we restrict the solution space of the pricing problems using problem specific knowledge. In our problem, it seems unlikely that in an optimal solution train units are coupled to (uncoupled from) the train, if on the next trip the expected number of passengers is lower (higher). Therefore, we initially forbid those transitions in the transition graphs of the trains. Next we apply the column generation procedure, until condition (17) is met. In a second phase, we allow all feasible 
transitions and apply the column generation procedure again. This two phase procedure considerably speeds up the convergence of the column generation algorithm. In our computational experiments, we observe a decrease in computation time for the LP relaxation of a factor 5 to 7 on average for larger instances compared to a single phase procedure.

\section{Branch-and-Price}

After solving the LP relaxation, branching is needed to obtain a proven optimal integer solution. Since only a subset of paths from $\Omega^{i}, i \in R$ is inserted in the master problem after solving the LP relaxation, and not necessarily all paths required for the optimal integer solution, the column generation procedure must be applied at every node of the branch-and-price tree with an appropriately modified pricing problem. These modifications to the pricing problem reflect the branching decisions taken at the current node, and the nodes higher in the tree on the path from the root node to the current node.

Our branching scheme consists of imposing and forbidding he deployment of a set of compositions on certain trips. More specifically, we build a binary search tree, where at the first node we impose that for a trip $t$ a composition must be chosen from a certain subset $C_{t}$ of the compositions $K_{t}$, whereas at the twin node a composition from $K_{t} \backslash C_{t}$ must be selected. Let $q_{t, k}^{\omega}=\sum_{j \in F_{t, k}} q_{t, k, j}^{\omega}$, as a result $q_{t, k}^{\omega}$. equals 1 , if trip $t$ uses composition $k$ in path $\omega$, and 0 otherwise, then the branching constraints are given by the following expression:

$$
\sum_{\omega \in \Omega^{i(t)}} \sum_{k \in C_{t}} q_{t, k}^{\omega} \lambda_{\omega}^{i(t)}=1 \text { and } \sum_{\omega \in \Omega^{i(t)}} \sum_{k \in C_{t}} q_{t, k .}^{\omega} \lambda_{\omega}^{i(t)}=0
$$

The branching constraints (18) need not be added explicitly to the master program, but can be implemented by deleting the appropriate columns from the master program (4), (6), (9)-(11) and by avoiding that these columns price out again by deleting nodes corresponding to forbidden compositions from the transition graph. We use a best first search strategy, i.e. we always branch from the node with the lowest lower bound. We now discuss the sufficient conditions to have an integer solution and next how we select the branching constraints.

Next, we argue that it is sufficient to impose that a combination, i.e. the number of train units of each material subtype, neglecting the order, is selected for each trip, and not necessarily a permutation in order to obtain an integer solution. Suppose for example that in the transition graph of 
Figure 4, we have imposed that on the trip between Asd and Rsd, the combination consisting of one unit of type "4" and one unit of type "6" must be used, corresponding to the compositions or permutations " 46 " and "64". Then, on the next trip between Rsd and Vs only two combinations and permutations can be used, i.e the composition that follows " 64 " namely " 4 " and the follower of " 46 ", namely "6". As a result, imposing a branching constraint for this following trip, e.g. use combination " 4 ", has the consequence that for the previous trip permutation " 64 " must be used. On the other hand, at the twin node, i.e. forbid combination " 4 ", combination " 6 " must be used on the Rsd-Vs trip and as a result permutation " 46 " between Asd and Rsd. In general, by branching on the combinations, all fractional permutations can be eliminated as well, and, as a consequence, the path through the transition graph is uniquely determined. However, there is one exception. It can happen, that there exist distinct paths through the transition graph of a train, although the combination is fixed for every trip. This is the case, if a train consists of a core component of at least two units of a different material subtype, that is deployed on all trips of the train during the day. Suppose, for instance, that a train consists of at least one unit of type "3" and one unit of type "4". During the day, other units can be coupled to and later uncoupled from this core component, to which two permutations correspond, i.e. "34" and "43". As a result, whatever train units are coupled or uncoupled, two paths through the transition graph do exist. Consequently, the composition is not uniquely defined. However, in this case, we can arbitrarily select one of those paths, and consider it as integer, since both are feasible and the relation with the other trains is identical for both paths, because the same units are (un)coupled after every trip. This insight allows to branch by imposing that a combination must be used for each trip rather than a permutation, which results in a smaller search tree and avoids symmetry.

The selection of the trip, for which we impose a branching constraint, is crucial in order to obtain an integer solution quickly. Based on the current master solution, we determine the fraction that a particular combination is used for each trip. From the set of trips for which there exists a fractional combination, we select the trip with the highest number of passengers and the combination with the largest fraction. The set of compositions $C_{t}$ of branching constraint (18) are then all permutations that correspond to the selected combination.

Finally, throughout the branch-and-price tree, we compute at every column generation iteration the lower bound on the LP value of equation (16). If this is greater than the best IP solution found, we can stop generating columns and prune the current node in our branch-and-price tree. 


\section{Numerical Experiments}

In order to test the described procedure, we carried out several computational experiments. The experiments are performed on an IBM NetVista 6343-25G Pentium 4 1.6 Ghz PC, using the Windows98 operating system. Our algorithm is coded in C++ and linked with the Extended LINDO/PC optimization library release 6.1 for solving the LPs.

\subsection{Description of the Data Sets}

Our procedure is tested on two cases, i.e. the 2100-2400-2600 train series presented in Table 1 and depicted in Figure 2, and the 3000 train series, that runs between Nijmegen (Nm) and Den Helder (Hdr) and is depicted in Figure 5. A 3000 train goes from Hdr to Alkmaar (Amr), where train units can be coupled or uncoupled. Then the train continues to Amsterdam (Asd), Utrecht (Ut), Arnhem (Ah) and Nijmegen. In Arnhem the train leaves the station in the same direction as it arrives. As a result, the composition is turned around such that the first train unit becomes the last and vice versa. This can be taken into account in the transition graphs, as explained earlier. In the terminal station $\mathrm{Nm}$, train units can be coupled or uncoupled as well. As a result, there are two stations where shunting operations can occur, i.e. Nm and Amr. Table 3 gives the number of trains, the number of trips during one day that must be performed, and the number of stations where (un)coupling can occur during the day.

Table 3: Data sets

\begin{tabular}{cccc}
\hline \hline Train series & Trips & Trains & Stations \\
\hline $2100-2400-2600$ & 182 & 15 & 5 \\
3000 & 115 & 12 & 2 \\
\hline
\end{tabular}

There exist three subtypes of train units, i.e. double deck units of three, four or six carriage, subsequently called DD3, DD4 and DD6. The maximum number of cars on each trip is 12, except for the trip Rsd-Vs where it is 10, and between Vs-Rsd, Amr-Hdr, and Hdr-Amr, where it is 9. For both series, we solve several instances by varying the availabilities of material subtypes of the train units and by varying the weights of the objective function.

\subsection{Computational Results}

Table 4 and Table 5 present the computational results. In Table 4, the weight given to a shunting operation, denoted as $w_{s}$, equals 0 , whereas in Table 5 , the weight equals 5 . The weight given to a kilometer-shortage is equal to 1 for a kilometer-shortage in second class $\left(w_{2}\right)$ and to 2 for a kilometershortage in first class $\left(w_{1}\right)$. The weight of a carriage-kilometer ( $\left.w_{\text {car }}\right)$ equals 0.01 . In our experiments, we relax the cyclicity constraint (6) and impose that the sum over the initial inventories of material 
subtypes in related station must be equal to the end inventory in those stations. We do this because the constraint (6) is too stringent, as explained earlier. In addition, in practice, there exist many ad hoc solutions to guarantee that the end inventory equals the initial inventory, which fall outside the scope of the current paper.

Figure 5: The 3000 Train Series

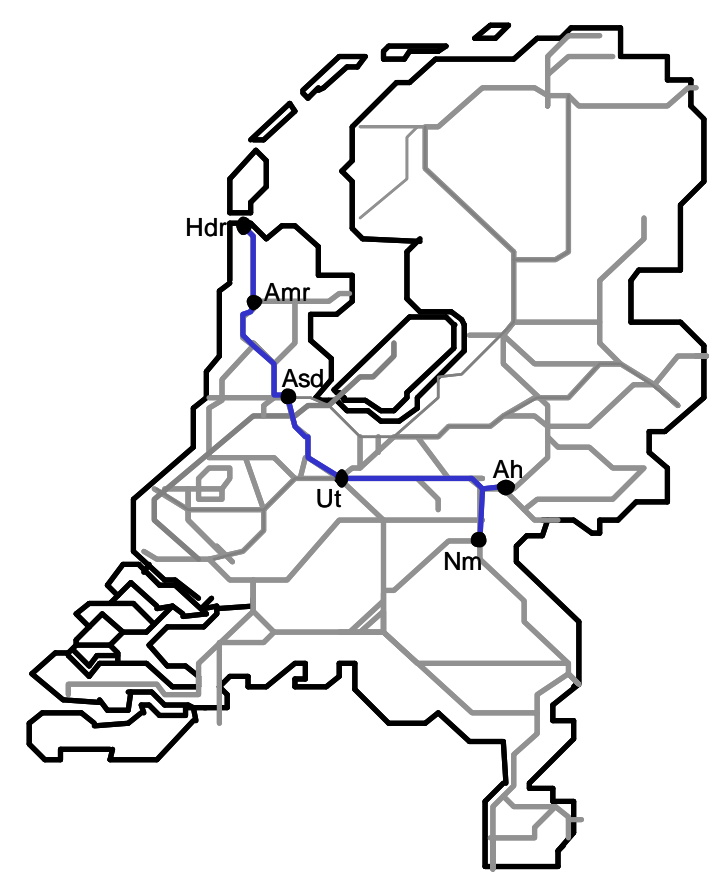

The column labelled "2000" (“3000”) gives the results for the train series 2100-2400-2600 (3000). The second row presents the number of material subtypes, i.e. 2 or 3 , and the number in brackets gives the number of instances solved for this data class, e.g. for the 3000 train series and three material subtypes, we solve 58 instances, by varying the availabilities of the different material subtypes. The remaining rows have the following meaning:

$T_{I P}: \quad$ Average CPU time to find the optimal integer solution (in seconds);

$T_{L P}$ : Average CPU time to find the LP relaxation at the root node (in seconds);

$n d s: \quad$ Average number of nodes in the branch-and-price tree;

$K_{L P}$ : Average number of generated columns at the root node;

$K_{I P}: \quad$ Average total number of generated columns;

$n c: \quad$ Average number of carriages;

gap: Average relative gap between the LP bound at the root node and the optimal solution, or the difference in IP solution and LP relaxation at the root node divided by the latter; 
CKM: Average number of carriage-kilometers;

S1: Average number of first class kilometer-shortages;

S2: Average number of second class kilometer-shortages;

SHT: Average number of shunting operations (i.e. coupling and uncoupling).

Based on Table 4 and 5, we can conclude that our procedure can solve all instances to optimality in reasonably short computation times. If the number of material subtypes equals 2 , our algorithm does not require more than 6 seconds on the average to obtain proven optimal solutions for the 2100-24002600 train series and less than a second for the 3000 train series. If the number of material subtypes equals 3, then the required CPU seconds increases to about one and a half minute for the 2100-24002600 train series and to about 10 seconds for the 3000 train series. The low average gap indicates that the LP lower bound is close to the optimal integer solution and, therefore, only few nodes must be investigated to obtain a proven optimal solution. We also did experiments with different weights assigned to the cost components. Since the computation time turned out to be insensitive to these changes, we opt for not reporting these results.

Table 4: Computational results.

\begin{tabular}{rrrrrr}
\hline \hline$w_{s}=0$ & \multicolumn{2}{c}{2000} & & \multicolumn{2}{c}{3000} \\
\cline { 2 - 3 } \cline { 6 - 7 }$T_{I P}$ & 5.86 & 91.27 & & 0.70 & 10.04 \\
$T_{L P}$ & 1.32 & 14.21 & & 0.35 & 1.13 \\
$n d s$ & 11.10 & 9.88 & & 10.89 & 17.52 \\
$K_{L P}$ & 304.61 & 574.34 & & 153.67 & 263.48 \\
$K_{I P}$ & 401.32 & 860.74 & & 184.11 & 443.69 \\
$n c$ & 145.47 & 155.49 & & 124.24 & 127.69 \\
$g a p$ & $0.92 \%$ & $0.68 \%$ & & $0.34 \%$ & $0.86 \%$ \\
$\mathrm{CKM}$ & 127171 & 133393 & & 113671 & 118617 \\
$\mathrm{~S} 1$ & 14.61 & 0.00 & & 104.49 & 24.21 \\
$\mathrm{~S} 2$ & 49095 & 23980 & & 142962 & 143120 \\
$\mathrm{SHT}$ & 78.68 & 77.12 & & 70.71 & 68.10 \\
\hline
\end{tabular}

Table 5: Computational results.

\begin{tabular}{rrrrrr}
\hline \hline \multirow{2}{*}{$w_{s}=5$} & \multicolumn{2}{c}{2000} & & \multicolumn{2}{c}{3000} \\
\cline { 2 - 3 } \cline { 6 - 7 }$T_{I P}$ & $4(62)$ & $3(82)$ & & $2(45)$ & $3(58)$ \\
$T_{L P}$ & 1.19 & 87.69 & & 0.60 & 9.84 \\
$n d s$ & 9.81 & 12.93 & & 0.36 & 1.17 \\
$K_{L P}$ & 305.11 & 566.82 & & 166.82 & 279.88 \\
$K_{I P}$ & 398.06 & 870.70 & & 197.44 & 462.24 \\
$n c$ & 145.47 & 155.49 & & 124.24 & 127.69 \\
$g a p$ & $0.90 \%$ & $0.67 \%$ & & $0.34 \%$ & $0.86 \%$ \\
$\mathrm{CKM}$ & 133231 & 138878 & & 117074 & 120520 \\
$\mathrm{~S} 1$ & 11.81 & 0.00 & & 104.49 & 24.21 \\
$\mathrm{~S} 2$ & 49101 & 23980 & & 142962 & 143120 \\
$\mathrm{SHT}$ & 53.39 & 55.39 & & 53.18 & 56.69 \\
\hline
\end{tabular}

\subsection{Comparison with CPLEX and with practice}

We compare the computation time of our branch-and-price procedure to formulation (1)-(8) solved by commercial software, in casu the modeling language OPL studio 3.6 using the CPLEX 7.0 solver. We compare our branch-and-price procedure for the same 58 instances of the 3000 train series with shunting weight equal to 0 and with three different material subtypes. The IP solution of both models is the same for all instances. The average CPU-time, however, is about 10 seconds for the branch-andprice algorithm (see Table 4) versus 227 seconds for CPLEX. We note that in CPLEX, we have 
imposed that the same branching priority is used as in the branch-and-price algorithm, and that integrality is enforced on the comb inations by introducing auxiliary variables.

For the 2100-2400-2600 train series, we were able to compare the circulation currently used in practice, with the circulation of our model. Actually, one DD3, 16 DD4's and 12 DD6's are deployed on the 2100-2400-2600 train series. Table 6 compares the circulation in practice with circulations proposed by the model for four scenarios. In the first two scenarios, we emphasize the minimization of the shortage-kilometers and use the same weights as for the experiments of Table 4 and 5 . In the next two scenarios, we give a relative higher weight to the carriage kilometers. We observe that the second class kilometer-shortages in practice are much higher than in the circulations proposed by our model. If we emphasize the carriage-kilometers, we are able to find circulations, with less carriages kilometers and a decrease in second class kilometer-shortages of about $65 \%$.

Table 6: Comparison between circulation in practice and solution of the model.

\begin{tabular}{|c|c|c|c|c|c|}
\hline & \multirow{2}{*}{$\frac{\text { emphasis: }}{\text { Practice }}$} & \multicolumn{2}{|c|}{ shortages ${ }^{(1)}$} & \multicolumn{2}{|c|}{ carriage $\mathrm{km}^{(2)}$} \\
\hline & & $w_{s}=0$ & $w_{s}=5$ & $w_{s}=0$ & $w_{s}=5$ \\
\hline CKM & 127521 & 130906 & 135084 & 125321 & 127860 \\
\hline S1 & 0 & 0 & 0 & 0 & 0 \\
\hline S2 & 90572 & 23583 & 23583 & 29981 & 27737 \\
\hline SHT & 60 & 77 & 58 & 76 & 62 \\
\hline
\end{tabular}

(2) $w_{1}=2, w_{2}=w_{1} / 2, w_{\mathrm{car}}=0.01$

We can conclude that our procedure is reasonably fast to perform several kinds of what-if analyses. As an illustration, we present two what-if studies in the next section.

\subsection{What-if Analyses}

For the 2100-2400-2600 train series, we investigate the effect of only using DD4 and DD6 on this line. We solve the circulation problem for every combination of DD4 and DD6 for which the total number of carriages is at least 120 and at most 180. A lower availability of carriages results in an infeasible solution and a higher availability in excess capacity. Figure 6 presents the results. On the Y-axis the total cost is given and on the X-axis the number of available DD6's. Every line on the chart presents the changes in the cost for a fixed number of DD4. We clearly observe that the marginal profit of an additional DD6 sharply decreases and quickly becomes 0. For example, for 3 DD4's, more than 26 units of DD6 cannot be deployed efficiently. We also observe that for the same number of carriages, the solution becomes more efficient if the share of DD4's increases. For example for 160 carriages, the total cost for 4 units of DD4 and 24 of DD6 is around 40,000, whereas the cost for 7 units of DD4 and 
22 units of DD6 is about 30,000 and even less than 20,000 for 10 units of DD4 and 20 of DD6.

Obviously, such results could have been guessed a priori, since units of DD4 are more flexible than units of DD6, due to their shorter length.

Figure 6: Circulation cost for the 2100-2400-2600 line (only units of 4 and 6 carriages)

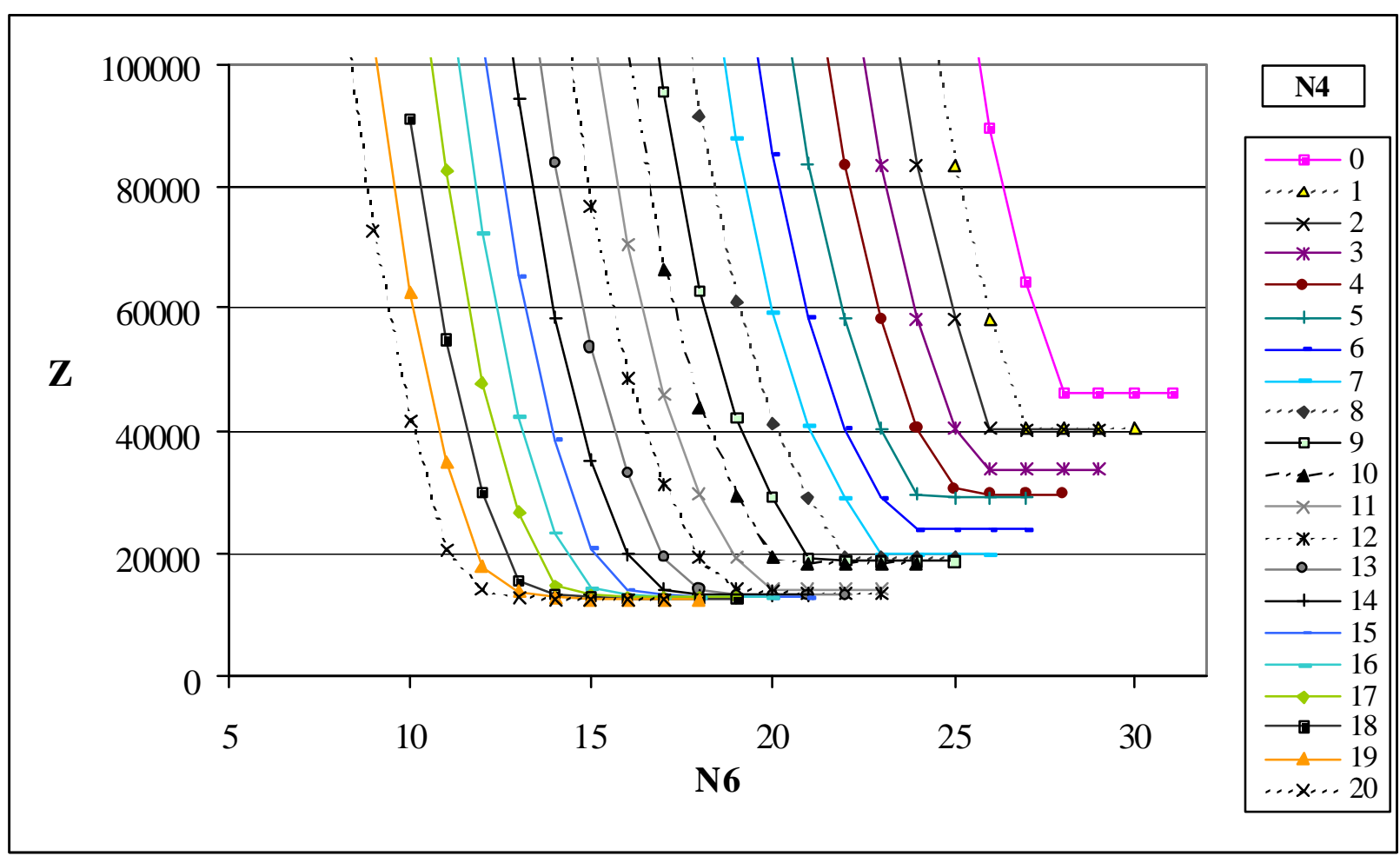

For the 3000 train series, we investigate the influence on the solution for two cases: (i) allowing coupling and uncoupling in Den Helder and (ii) increasing in the maximum train length from 9 to 12 carriages, between Amr and Hdr and vice versa. In case (i), the number of trips increases then from 115 to 148 . Table 7 presents a comparison for both cases with the original situation, called "baseline". The column labelled " $m t$ " gives the number of material subtypes. The entries in the Table are the averages over the same instances as in Tables 4 and 5. The average computation time for case (i) increases to about 1 minute for the instances with 3 material subtypes, due to the higher number of trips. We observe that there is no effect on the average first class kilometer-shortages and that the effect on the second class kilometer-shortages is insignificant, i.e. about $0.02 \%$ for $m t=2$ and $0.05 \%$ for $m t=3$. The reductions in carriage-kilometers is very small, if a positive weight is given to the shunting operations. If not, a reduction of $1 \%$ can be achieved if two material subtypes are used and $0.5 \%$ if three subtypes are used. As a result, an additional shunting possibility in Hdr does not seem to have a big impact on the solution quality. Increasing the maximum train length to 12 carriages, however, results in a 
decrease of the kilometer-shortages with about $10 \%$ and an increase in carriage-kilometers with about $2 \%$. It can be concluded that the limited maximum train length of 9 carriages is indeed a bottleneck.

Table 7: Cases for the 3000 Train series

\begin{tabular}{|c|c|c|c|c|c|c|c|}
\hline & $m t$ & case & CKM & S1 & $\mathrm{S} 2$ & SHT & $T_{I P}$ \\
\hline \multirow[t]{6}{*}{$w_{s}=0$} & 2 & baseline & 113671 & 104.5 & 142962 & 70.7 & 0.70 \\
\hline & & (i) Sht in Hdr & 112526 & 104.5 & 142933 & 79.0 & 2.29 \\
\hline & & (ii) $\max 12$ & 114084 & 97.5 & 125505 & 65.6 & 0.73 \\
\hline & 3 & baseline & 118617 & 24.2 & 143120 & 68.1 & 10.04 \\
\hline & & (i) Sht in $\mathrm{Hdr}$ & 117934 & 24.2 & 143044 & 71.5 & 62.46 \\
\hline & & (ii) $\max 12$ & 120123 & 28.6 & 125206 & 61.7 & 12.27 \\
\hline \multirow[t]{6}{*}{$w_{s}=5$} & 2 & baseline & 117074 & 104.5 & 142962 & 53.2 & 0.60 \\
\hline & & (i) Sht in Hdr & 116944 & 104.5 & 142933 & 53.3 & 1.64 \\
\hline & & (ii) $\max 12$ & 119048 & 97.5 & 125505 & 42.4 & 0.67 \\
\hline & 3 & baseline & 120521 & 24.2 & 143120 & 56.7 & 9.84 \\
\hline & & (i) Sht in $\mathrm{Hdr}$ & 120462 & 24.2 & 143044 & 56.8 & 61.13 \\
\hline & & (ii) $\max 12$ & 123722 & 28.6 & 125206 & 43.7 & 16.95 \\
\hline
\end{tabular}

\section{Conclusions and Future Research}

We have presented a model to determine an efficient rolling stock circulation for a set of interacting train series. We have developed a branch-and-price algorithm to solve the problem to a proven optimal solution. The algorithm is tested on real life instances from NS Reizigers, the main Dutch operator of passenger trains. The required CPU time is short, as follows from a comparis on with CPLEX. Therefore, what-if analyses can be performed easily. The model can be a useful tool for planners to determine the number of material subtypes allocated to a line. In addition, the circulation proposed by the model can be a good starting point to make the real circulation plan. However, this will require the availability of a user-friendly graphical user-interface in addition to our algorithmic approach.

In our future research, we will focus on the application of the proposed procedure to the splitting and combining of trains. Indeed, it may happen that a train is split into two trains at a station, leaving in different directions, and that two trains coming from different directions arrive in a station and are combined into one train. We will investigate how the model and the algorithmic approach can be adapted to include this issue. Also other complicating issues, such as a larger number of material types and subtypes per train series, will be a subject for our future research.

\section{Acknowledgement}

The authors are partly sponsored by the Human Potential Program of the European Union under contract no. HPRN-CT-1999-00104 (AMORE). 


\section{References}

AbBink, E., B. VAN DEN BerG, L. KROON AND M. SAlOMON. 2003. Allocation of Railway Rolling Stock for Passenger Trains. Transportation Science, to appear. ERIM Research Report, ERS2002-47-LIS. Erasmus University Rotterdam, The Netherlands.

Ahuja, R.K., T.L. Magnanti, AND J.B. ORLIN. 1993. Network Flows: Theory, Algorithms, and Applications. Prentice-Hall, Englewood Cliffs, NJ.

Alfieri, A., R. GROOT, L. KROON AND L. SCHRIJVER. 2002. Efficient Circulation of Railway Rolling Stock. ERIM Research Report, ERS-2002-110-LIS. Erasmus University Rotterdam, The Netherlands.

BARnhart, C., E.L Johnson, L.E. NEMHAuser, M.W.P. SA Velsbergh AND P.H. VANCE. 1998. Branch-and-Price: Column Generation for Solving Huge Integer Programs. Operations Research 43, 316-329.

BARNHART, C., C.A: HANE AND P.H. VANCE. 2000. Using Branch-and-Price-and-Cut to Solve OriginDestination Integer Multicommodity Flow Problems. Operations Research 48, 318-326.

Ben-Khedher, N., J. Kintanar, C. QueILle, AND W. Stripling. 1998. Schedule Optimization at SNCF: From Conception to Day of Departure. Interfaces 28, 6-23.

BruCKer, J., T. Hurink AND T. ROLFES. 1998. Routing of Railway Carriages: A Case Study. Osnabrücker Geschriften zur Mathematik, Reihe P, Heft 205.

Cordeau, J.F., F. SOUMIS, AND J. DeSROSIERS. 2000. A Benders Decomposition Approach for the Locomotive and Car Assignment Problem.. Transportation Science 34, 133-149.

CORDEAU, J.F., F. SOUMIS, AND J. DESROSIERS. 2001. Simultaneous Assignment of Locomotives and Cars to Passenger Trains. Operations Research 49, 531-548.

DANTZIG, G.B. AND P. WOLFE. 1960. Decomposition Principle for Linear Programming. Operations Research 8, 101-111.

DeSROSIERS, J., AND F. SOUMIS. 1989. A Column Generation Approach to the Urban Transit Crew Scheduling Problem. Transportation Science 23, 1-12.

Desrosiers, J., F. SOUMIS AND M. DESROCHERS. 1984. Routing with Time Windows by Column Generation. Networks 14, 545-565.

HolmberG, K. AND D. YUAN. 2003. A Multicommodity Network Flow Problem with Side Constraints on Paths Solved by Column Generation. Informs Journal on Computing 15, 42-57. 
Lingaya, N., J.F. Cordeau, G. DeSAulniers, J. DeSRosiers, AND F. SOUMIS. 2002. Operational Car Assignment at VIA Rail Canada. Transportation Research B 36, 755-778.

SAVElsBerGH, M. 1997. A Branch-and-Price Algorithm for the Generalized Assignment Problem. Operations Research 45, 831-841.

SCHRIJVER, A. 1993. Minimum Circulation of Railway Stock. CWI Quarterly 6, 205-217.

VANDERBECK, F. 2000. On Dantzig-Wolfe Decomposition in Integer Programming and Ways to Perform Branching in a Branch-and-Price Algorithm. Operations Research 48, 111-128

VANDERBECK, F. AND L.A. WOLSEY 1996. An Exact Algorithm for IP Column Generation. Operations Research Letters 19, 151-159.

WOLSEY, L.A. 1998. Integer Programming. Wiley Interscience Series in Discrete Mathematics and Optimization, John Wiley \& Sons, Chichester. 


\section{Publications in the Report Series Research* in Management}

ERIM Research Program: "Business Processes, Logistics and Information Systems"

2003

Project Selection Directed By Intellectual Capital Scorecards

Hennie Daniels and Bram de Jonge

ERS-2003-001-LIS

http://hdl.handle.net/1765/265

Combining expert knowledge and databases for risk management

Hennie Daniels and Han van Dissel

ERS-2003-002-LIS

http://hdl.handle.net/1765/266

Recursive Approximation of the High Dimensional max Function

Ş. II. Birbil, S.-C. Fang, J.B.G. Frenk and S. Zhang

ERS-2003-003-LIS

http://hdl.handle.net/1765/267

Auctioning Bulk Mobile Messages

S.Meij, L-F.Pau, E.van Heck

ERS-2003-006-LIS

http://hdl.handle.net/1765/274

Induction of Ordinal Decision Trees: An MCDA Approach

Jan C. Bioch, Viara Popova

ERS-2003-008-LIS

http://hdl.handle.net/1765/271

A New Dantzig-Wolfe Reformulation And Branch-And-Price Algorithm For The Capacitated Lot Sizing Problem With Set Up Times

Zeger Degraeve, Raf Jans

ERS-2003-010-LIS

http://hdl.handle.net/1765/275

Reverse Logistics - a review of case studies

Marisa P. de Brito, Rommert Dekker, Simme D.P. Flapper

ERS-2003-012-LIS

http://hdl.handle.net/1765/277

Product Return Handling: decision-making and quantitative support

Marisa P. de Brito, M. (René) B. M. de Koster

ERS-2003-013-LIS

http://hdl.handle.net/1765/278

\footnotetext{
A complete overview of the ERIM Report Series Research in Management: http://www.erim.eur.nl

ERIM Research Programs:

LIS Business Processes, Logistics and Information Systems

ORG Organizing for Performance

MKT Marketing

F\&A Finance and Accounting

STR Strategy and Entrepreneurship
} 
Managing Product Returns: The Role of Forecasting

Beril Toktay, Erwin A. van der Laan, Marisa P. de Brito

ERS-2003-023-LIS

http://hdl.handle.net/1765/316

Improved Lower Bounds For The Capacitated Lot Sizing Problem With Set Up Times

Zeger Degraeve, Raf Jans

ERS-2003-026-LIS

http://hdl.handle.net/1765/326

In Chains? Automotive Suppliers and Their Product Development Activities

Fredrik von Corswant, Finn Wynstra, Martin Wetzels

ERS-2003-027-LIS

http://hdl.handle.net/1765/363

Mathematical models for planning support

Leo G. Kroon, Rob A. Zuidwijk

ERS-2003-032-LIS

http://hdl.handle.net/1765/332

How and why communications industry suppliers get "squeezed out" now, and the next phase

L-F Pau

ERS-2003-033-LIS

http://hdl.handle.net/1765/317

Financial Markets Analysis by Probabilistic Fuzzy Modelling

Jan van den Berg, Uzay Kaymak, Willem-Max van den Bergh

ERS-2003-036-LIS

http://hdl.handle.net/1765/323

WLAN Hot Spot services for the automotive and oil industries :a business analysis or : "Refuel the car with petrol and information , both ways at the gas station "

L-F Pau, M.H.P.Oremus

ERS-2003-039-LIS

http://hdl.handle.net/1765/318

A Lotting Method for Electronic Reverse Auctions

U. Kaymak, J.P. Verkade and H.A.B. te Braake

ERS-2003-042-LIS

http://hdl.handle.net/1765/337

Supply Chain Optimisation in Animal Husbandry

J.M. Bloemhof, C.M. Smeets, J.A.E.E. van Nunen

ERS-2003-043-LIS

http://hdl.handle.net/1765/353

A Framework for Reverse Logistics

Marisa P. de Brito and Rommert Dekker

ERS-2003-045-LIS

http://hdl.handle.net/1765/354

An assessment system for rating scientific journals in the field of ergonomics and human factors Jan Dul and Waldemar Karwowski

ERS-2003-048-LIS

http://hdl.handle.net/1765/432 